TAO, Vol. 13, No. 1, 1-14, March 2002

\title{
A Study of Early High-frequency Precursors by Physical Modeling
}

\author{
Young-Fo Chang ${ }^{1, *}$ and Bor-Shouh Huang ${ }^{2}$
}

(Manuscript received 24 September 2001, in final form 25 December 2001)

\begin{abstract}
Early high-frequency precursors, which arrive before the onset of $\mathbf{P}$ waves, have been observed at some subducted zones. Seismologists considered this phenomenon to be the result of high-frequency seismic waves propagation in a high-velocity layer (HVL). Although some numerical simulations have been used to explain the existence of early high-frequency precursors, a high frequency 3-D elastic model has not been considered.

In this high frequency 3-D physical modeling study, thin duralumin plates of different thicknesses, embedded in epoxyblocks, were used to study the elastic wave propagation in a thin HVL. The experimental results reveal that high-velocity and high-frequency precursors can be generated using a thin HVL model, and the high-frequency precursors arrive earlier than low-frequency ones. It was also observed that the thinner the HVL, the smaller the amplitudes and higher the frequency of the precursors. Based on the travel times and amplitudes of the precursors, it can be concluded that P-to-P ( $P$ converts to $P$ ) interactions play a major role in controlling the propagation of precursors. The precursors are the $P$ waves resonance in the HVL; therefore, the curvature and the period of the holes (the local low values) in the dispersion curve may be used to estimate the thickness of the HVL.
\end{abstract}

(Key words: Thin high-velocity layer, Early high-frequency precursors, Physical model)

\section{INTRODUCTION}

Early high-frequency precursors arriving prior to the onset of the direct $\mathrm{P}$ waves have been observed at the Vanuatu (Chiu et al. 1985), Taiwan (Shin 1992), and Tonga-Kermadec

\footnotetext{
${ }^{1}$ Institute of Applied Geophysics, Institute of Seismology, National Chung Cheng University, Min-hsiung, Chia-yi, Taiwan, ROC

${ }^{2}$ Institute of Earth Sciences, Academia Sinica, Taiwan, ROC

${ }^{\star}$ Corresponding author address: Prof. Young-Fo Chang, Institute of Applied Geophysics, Institute of Seismology, National Chung Cheng University, Min-hsiung, Chia-yi 621, Taiwan, ROC

E-mail: seichyo@eq.ccu.edu.tw
} 
subduction zones (Ansell and Gubbins 1986; Gubbins and Snieder 1991; Galea 19922; Smith et al. 1994; Stuart et al. 1995; Van der Hilst and Snieder 1996). The mechanism of these anomalous phases has been inferred to be short-wavelength waves traveling within a thin high-velocity layer (HVL), while the longer wavelengths are not affected by the HVL and ravel at normal mantle speed. The thin HVL is interpreted as an eclogite layer in which subduct ed oceanic crust is changed with high-pressure and high-temperature conditions (Gubbins et al. 1994).

Numerical simulations have been used to model the early high-frequency precursors. The arrival times of these early high-frequency precursors, relative to the Jeffreys-Bullen travel time, were used to explain how the precursors result from the waves refracted from the thin HVL using the ray method (Huppert and Frohlich 1981; Chiu et al. 1985; Ansell and Gubbins 1986; Galea 1992). Smith et al. (1994) analyzed the travel times and polarizations of the early high-frequency precursors, and concluded that the early high-frequency precur-sors travel through a high-velocity layer close to the top of the subducted plate and refract upsward to the station.

Gubbins and Snieder (1991) modeled the dispersion of the early high-frequerncy precursors as acoustic waves propagation within a 1-D slab, and calculated the waveform.s using the reflectivity method. They concluded that the early high-frequency precursors can exist within 2-layer or ramp models. Van der Hilst and Snieder (1996) modeled the waveforms of the early high-frequency precursors in 2-D models using a parabolic approximation, and con cluded that the characteristics of the early high-frequency precursors depend upon the locat ions of the source, the receiver and the HVL structure. These studies have been successful in explaining the essential attributes of the precursory phases.

The HVL is so thin that ray theory used to model the early high-frequency prec-ursors may break down and frequency dependent wave propagation may occur (Gubbins a.nd Snieder 1991). Although both Gubbins and Snieder (1991) and Van der Hilst and Snieder (1996) employed acoustic wave methods to model the precursors, which imply that the major role in controlling the propagation of precursors is the result of P-to-P interactions. A higla frequency 3-D elastic model for studying the early high-frequency precursors is still ne eded to be considered.

Since the mechanisms of seismic and ultrasonic wave propagation are the same, we can infer the characteristics of seismic waves from the results obtained from the expe riments for the ultrasonic waves. Therefore, in this study a high frequency 3-D elastic model (physical modeling) is used to study the early high-frequency precursors. Two different wa ve types (P wave and $\mathrm{S}$ wave) and four different frequency $\mathrm{P}$ wave transducers were used t o study the essential characteristics of the precursors propagation in three HVLs of different t.hicknesses.

\section{EXPERIMENTS}

Ultrasonic high frequency 3-D elastic models were used to examine the ear 1 high-frequency precursors in this study. The basic principle of the acquisition system for the physical modeling experiment is the same as that of the common ultrasonic nondestructive evaluation system. The two-probe pulse-echo transmission technique was used in this experiment. The pulser-receiver (Panametrics 5058PR) generates a high-voltage pulse to excite the sourcetransducer, which receives and amplifies the low-voltage signal detected by the receiver- 
ransducer. The source-transducer converts the electrical energy (high-voltage pulse) into elastic energy (ultrasonic waves), and the receiver-transducer transforms elastic energy back into electrical energy. Five kinds of contact transducers ${ }^{\mathrm{TM}}$ (Table 1) were used in this study, including four different dominant frequencies, two different wave types ( $\mathrm{P}$ and $\mathrm{S}$ waves) and two different frequency bands (narrow band and broad band). The $\mathrm{P}$ and $\mathrm{S}$ wave transducers were used to detect (or radiate) the particle motion of the ultrasound perpendicular to and

Table 1. The $\mathrm{P}$ and $\mathrm{S}$ waves transducers ${ }^{\mathrm{TM}}$ used in experiments.

\begin{tabular}{|c|c|c|c|c|c|}
\hline Transducers & Model & $\begin{array}{c}\text { Diameter } \\
\text { (inch) }\end{array}$ & $\begin{array}{c}\text { Frequency } \\
\text { (MHz) }\end{array}$ & Wave type & $\begin{array}{c}\text { Frequency } \\
\text { band }\end{array}$ \\
\hline T1 & Panametrics V103 & 0.5 & 1 & P wave & Broad band \\
\hline T2 & Panametrics A113S & 0.25 & 2.5 & P wave & Narrow band \\
\hline T3 & Panametrics A109S & 0.5 & 5 & P wave & Narrow band \\
\hline T4 & Panametrics A111S & 0.5 & 10 & P wave & Narrow band \\
\hline T5 & Ultran SWC18-5 & 0.25 & 5 & S wave & Broad band \\
\hline
\end{tabular}

parallel to the ransducer faces, respectively. The transducer cannot be put into the model and can only be coupled to the surface of the model. A digital oscilloscope (Tektronix TDS420) is used to monitor the signals and convert the analog signal into a digital signal. A personal computer then reads the digital signals from the oscilloscope, and processes the signals. The apparatus configuration is shown in Fig. 1. The sampling rate of the digital oscilloscope was set to 0.02 micro second and 5120 points per trace were recorded.

This study does not seek to model the actual velocity structure of the Earth nor to match precisely the actual waveforms of the early high-frequency precursors. Rather, our objective is to investigate the structural conditions specific to the existence of the early high-frequency precursors, and to analyze the general characteristics of the early high-frequency precursors. Early high-frequency precursors are heavily attenuated and can never be observed at teleseismic distances due to the energy leakage out of the HVL (Gubbins and Snieder 1991). Therefore, in order to get enough separation between the travel times and to increase the difference in am-

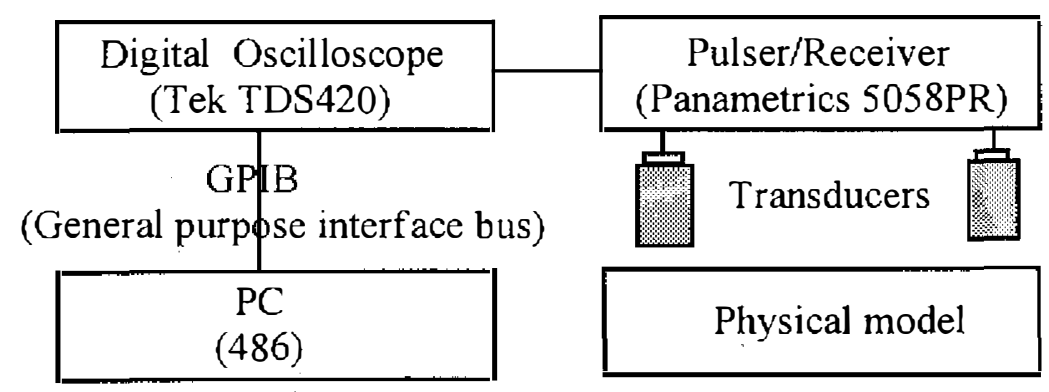

Fig. 1. The configuration of the apparatus used. 
plitude between the precursors and the normal $\mathrm{P}$ waves during a small propagation distance, the thin duralumin plate (thickness 1, 3, $5 \mathrm{~mm}$ ) and epoxy (Kuo-Sen EP231) materials with strong velocity and attenuation contrast, were used to simulate the HVL and mantle, respectively. The source is coupled to the thin HVL, and receivers are deployed on the opposite surface of the model, shown in Fig. 2. The densities and velocities of the epoxy and the duralumin plate, which we measured, are shown in Table 2.

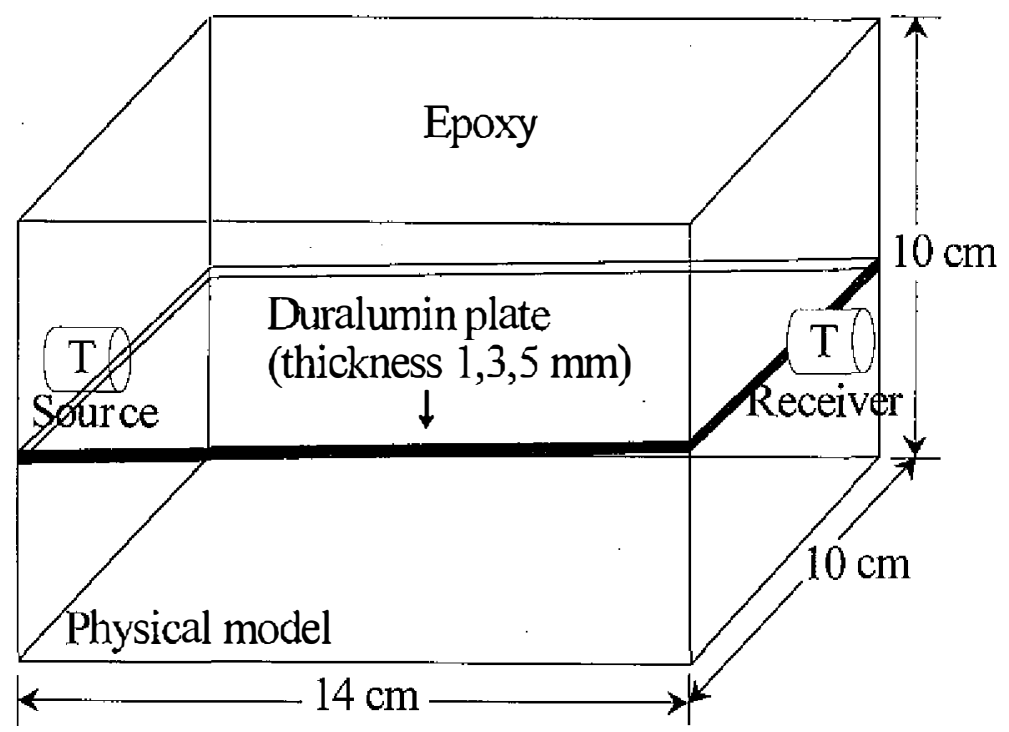

Fig. 2. The configuration of the model. There are three models with three different thickness $(1,3,5 \mathrm{~mm})$ duralumin plates, embedded at a depth of $5 \mathrm{~cm}$ in the epoxy. The source is located on the duralumin plate, and the receiver is also deployed on the duralumin plate and on the opposite surface of the model.

\section{RESULTS}

The source and receiver were located on the opposite surfaces of the model, as shown in Fig. 2. When the thickness of the thin duralumin plate is $1 \mathrm{~mm}$ (model Ml), the echoes emitted and recorded by pair $1 \mathrm{MHz}$ broad band transducers (T1) and by the $2.5,5$ and $10 \mathrm{MHz}$ narrow

Table 2. The velocities and densities of the epoxy and duralumin plate used in the experiments.

\begin{tabular}{|c|c|c|c|}
\hline Medium & \multirow{2}{*}{\begin{tabular}{l} 
Density \\
\cline { 3 - 4 }
\end{tabular}} & \multicolumn{2}{|c|}{ Velocity (m/s) } \\
\cline { 3 - 4 } & $\left(\mathrm{g} / \mathrm{cm}^{3}\right)$ & P waves & S waves \\
\hline Epoxy & 1.1 & 2381 & 1054 \\
\hline Duralumin & 2.7 & 6242 & 3100 \\
\hline
\end{tabular}


band transducers (T2, T3 and T4) are shown in Fig. 3. The response curve of the transducer in the frequency domain is bell-shaped and the maximum response is located at the resonant frequency of the transducer. After coupling with the model, however, the response is not easy to estimate. Therefore, the amplitude scales of the traces in Fig. 3 are arbitrary. The high frequency echo attenuates more rapidly than the low-frequency one; consequently, a larger amplification factor was applied to the top trace, and the background noise therefore is also amplified. The slashed line in the figure is the arrival time of $P$ wave propagation at $P$ wave velocity in the epoxy. The waves designated as ' $\mathrm{P}$ ' propagate within the epoxy, and are analo-

\section{M1 (Thinckness 1mm)}

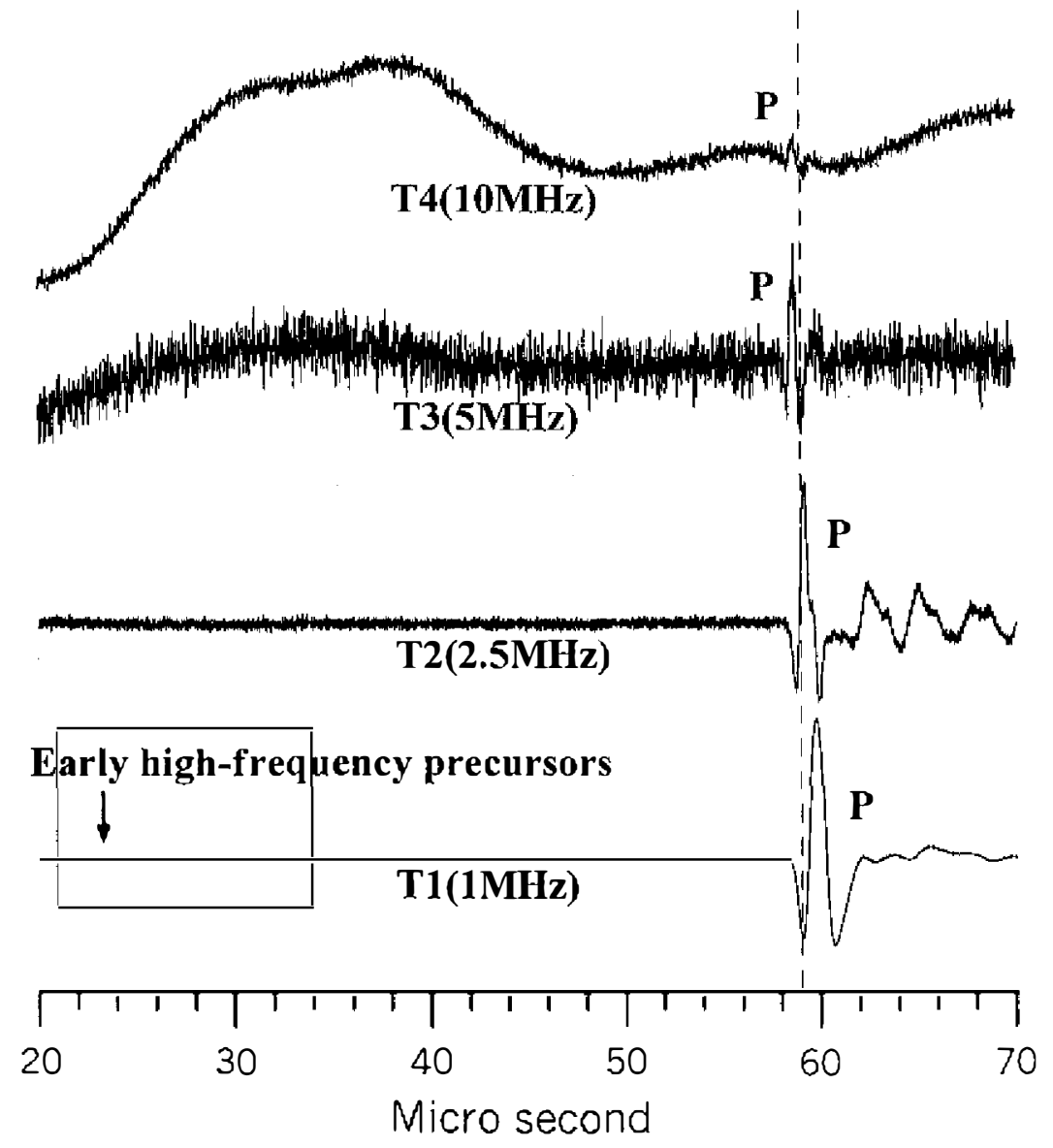

Fig. 3. The traces recorded on the model with $1 \mathrm{~mm}$ thickness duralumin plate. The amplitude scales of the traces are arbitrary. The waves designated ' $\mathrm{P}$ ' are the $\mathrm{P}$ waves propagation within the epoxy. The slashed line is the arrival time of $\mathrm{P}$ wave propagation at $\mathrm{P}$ wave velocity in the epoxy, and the time indicated by the arrow in the bottom race is the arrival time of $P$ wave propagation at $\mathrm{P}$ wave velocity in the thin duralumin plate. 
gous to the wave propagation in normal mantle. The time indicated by the arrow in the bottom trace is the arrival time of $P$ wave propagation at $P$ wave velocity in the thin duralumin plate. The higher frequency waves, travel at a higher velocity within the duralumin and are evident only in the bottom trace. These waves are analogous to the early high-frequency precursors refracted from the HVL. Unfortunately the amplitude level of the waves approached the resolution limit of the apparatus and was too weak for further analysis.

The traces recorded in the models with thicknesses of $3 \mathrm{~mm}$ (M2) and $5 \mathrm{~mm}$ (M3) duralumin plates by the same types of transducers are shown in Figs. 4 and 5, respectively. The slashed line is the arrival time of $\mathrm{P}$ wave propagation at $\mathrm{P}$ wave velocity in the thin duralumin

M2 (Thinckness 3mm)

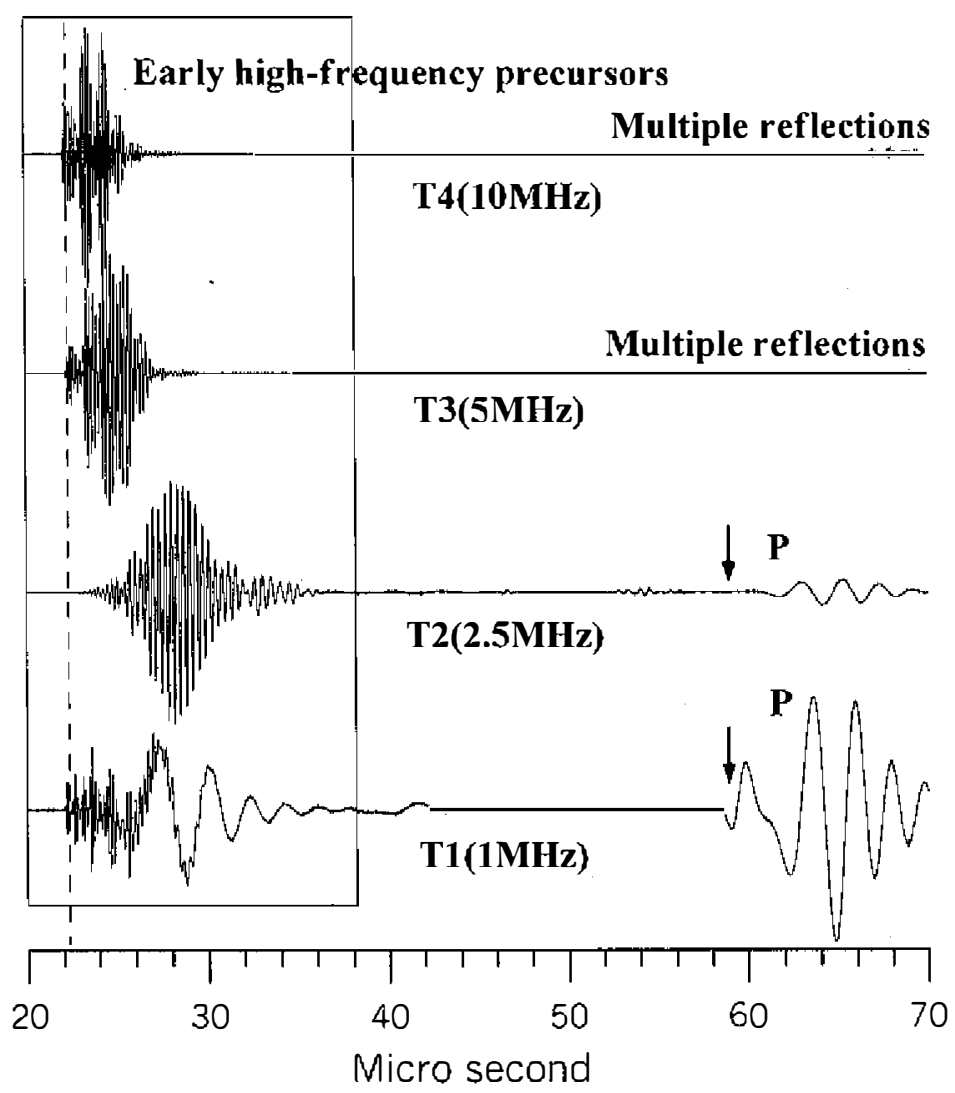

Fig. 4. The traces recorded on the model with $3 \mathrm{~mm}$ thickness duralumin plate. The amplitude scales of the traces are arbitrary. The waves designated ' $\mathrm{P}$ ' are the $\mathrm{P}$ waves propagation within the epoxy. The slashed line is the arrival time of $\mathrm{P}$ wave propagation at $\mathrm{P}$ wave velocity in the thin duralumin plate, and the times indicated by the arrows are the arrival times of $P$ wave propagation at $\mathrm{P}$ wave velocity in the epoxy. 
M3 (Thinckness 5m m)

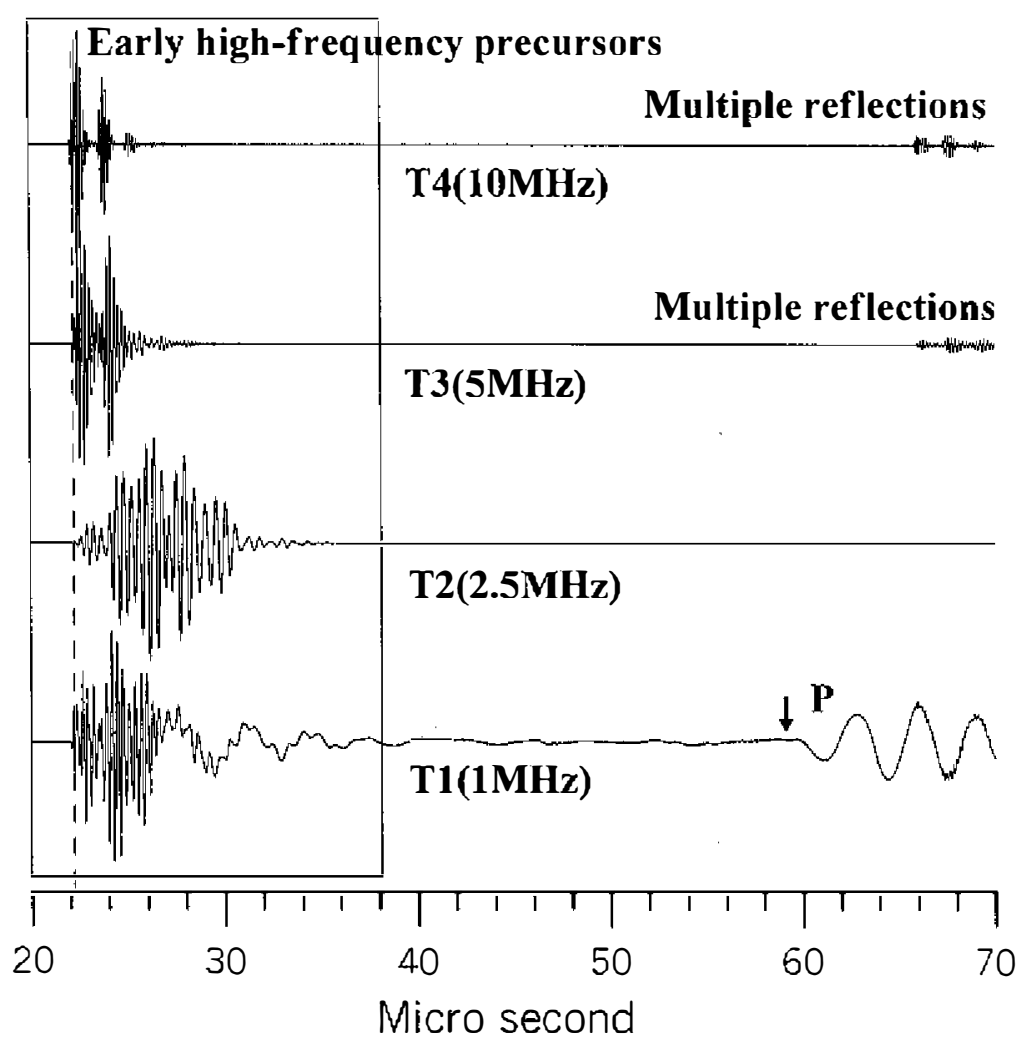

Fig. 5. The traces recorded on the model with $5 \mathrm{~mm}$ thickness duralumin plate. The amplitude scales of the traces are arbitrary. The waves designated ' $\mathrm{P}$ ' are the $\mathrm{P}$ waves propagation within the epoxy. The slashed line is the arrival time of $P$ wave propagation at $P$ wave velocity in the thin duralumin plate, and the time indicated by the arrow in the bottom trace is the arrival time of $\mathrm{P}$ wave propagation at $\mathrm{P}$ wave velocity in the epoxy.

plate and the times indicated by the arrows are the arrival times of $P$ wave propagation at $P$ wave velocity in the epoxy. In both figures, the early high-frequency precursors can be clearly seen. The amplitudes of the early high-frequency precursors are small when compared to the normal arrival P phases (Chiu et al. 1985; Ansell and Gubbins 1986; Smith et al. 1994; Stuart et al. 1995). On the other hand, in our experiments they are large due to the high attenuation of the normal $P$ waves by the epoxy. The narrow band transducer can only emit and record the echoes close to its dominant frequency. The precursors recorded by the narrow band transducers (T2-T4) show that different frequency precursors arrive at different times with the highfrequency waves arriving earlier than the low-frequency ones, and the precursors in Model M3 arriving earlier than the ones in Model M2. The bottom traces (in both figures) produced by the broad band transducers (T1) show that the different frequency precursors which arrive 
at different times on the traces; high-frequency waves arrive earlier than the low-frequency waves. The frequency of the precursors in the Model M2 is also higher than that in the Model M3.

The ultrasonic energy is almost introduced into the epoxy when the diameter of the transducer is larger than the thickness of the HVL, therefore the normal ' $\mathrm{P}$ ' waves can be observed in all traces of Fig. 3. On the other hand, the greater part of ultrasonic energy is introduced into the duralumin plate and the normal ' $\mathrm{P}$ ' wave can only be observed on low frequency trace (in T2 of Fig. 4) or broad band (Figs. 4 and 5) traces.

The time windows, which contain the early high-frequency precursors in Figs. 4 and 5, are truncated, and the corresponding Fourier amplitude spectra are shown in Fig. 6. The local maxima in the spectra appear periodically with a periodicity for the M2 and M3 models of 1 . 1 and $0.7 \mathrm{MHz}$, respectively. The local maximum frequencies can be considered as the resonant frequencies of the waves propagation within the thin HVL. The dominant wavelengths of the local maximum frequencies are equal to $2 t / n$, where $n$ is an integer indicating the order of the harmonic and $t$ is the thickness of the HVL. This is like a half-wavelength resonance when the phase changes on the reflection are the same at both the ends of a body (Blitz and Simpson 1996).

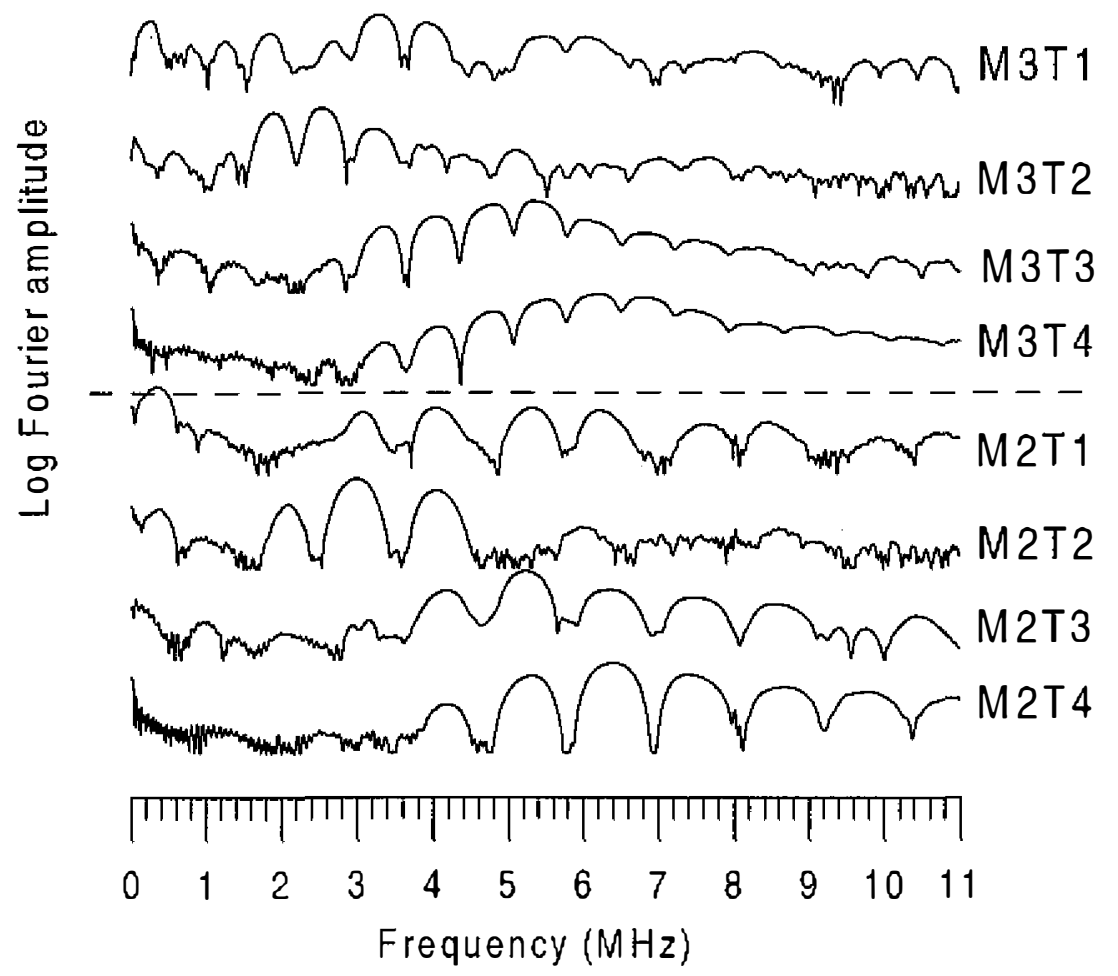

Fig. 6. The Fourier amplitude spectra of the precursors in Figs. 4 and 5. The bottom trace (M2T4) is the signal recorded by the T4 transducers on Model M2, trace M2T3 is the signal recorded by the T3 transducers on Model M2, and so on. 
A 1.2 micro second time window is moved along the time axis, and the Fourier amplitude spectrum of the signal in the time window is calculated. After averaging and smoothing the Fourier amplitude spectra along the time axis, the dispersion of the precursors is analyzed. The dispersion curves of the precursors recorded on the M2 and Model M3s are shown in Figs. 8 and 9, respectively. The signal emitted and detected by the broad band transducers is distributed over a broad frequency range. However, both figures show that the high-frequency waves arrive earlier than the low-frequency ones. The curvatures of dispersion curves are different in Figs. 8 and 9 for the different thickness HVLs. The curvature of dispersion curve in the $3 \mathrm{~mm}$ thickness model (Fig. 7) is smoother than that in the $5 \mathrm{~mm}$ thicleness model (Fig. 8). Therefore, the curvature of dispersion curve of the precursors can be used to estimate the thickness of the thin HVL.

The echoes detected by the S-type receiver (T5) on the Model M2 for the angles $(\theta) 0^{\circ}$ $90^{\circ}$ (in increments of $30^{\circ}$ ) are shown in Fig. 9. The polarizations $0^{\circ}$ and $90^{\circ}$ of $\mathrm{S}$ wave transducer are parallel to and perpendicular to the HVL, respectively. The long and short slashed lines are the arrival times of $P$ and $S$ waves propagation at $P$ and $S$ wave velocities in the thin duralumin plate, respectively. The most interesting events in Fig. 9 are the P-to-S type early high-frequency precursors propagation at the $S$ wave velocity in the thin HVL. The frequencies of the P-to-S type early high-frequency precursors change from low to high with the decrement of $\theta$. This is because the $S$ waves must resonate in the thin HVL for the particle motions perpendicular to HVL. But, the particle motion of the $S$ wave parallel to the HVL does not resonate, and the dominant frequency of the wave is $1 \mathrm{MHz}$ that is the dominant frequency of the wave radiated from the source T2. We assume that the levels of background noise are the same during the experiments. After comparing the background noises in Figs. 4 and 5 with those in Fig. 9, the result that the traces in Fig. 9 are enlarged more than that in Figs. 4 and 5 can be obtained. Consequently, the amplitude of the precursors whose polarizations are parallel to the wave direction is larger than those whose are perpendicular to the wave direction. Thus, the P-to-P (P converts to P) type acoustic models (Gubbins and Snieder 1991; Van der Hilst and Snieder 1996) have been successful in explaining the essential attributes of the precursory phases.

\section{DISCUSSIONS}

Real earth structures are more complex than our models and both 2-layer and ramp models (Gubbins and Snieder 1991); thus, undulating slab and lateral variation in thickness models (Van der Hilst and Snieder 1996) have been proposed. Also, Gubbins and Snieder (1991) concluded that the uniform slab can produce the required dispersion when the slab is thin enough; however, the attenuation associated with energy leakage away from the slab is so great that the waves would never be observed. Our object in using the uniform slab model is neither to simulate the actual earth structure nor to synthesize the waveforms of the observed early high-frequency precursors for subduction zones. But, studying the origin and characteristics of the early high-frequency precursors is emphasized in this study.

Earlier researchers inferred that the early high-frequency precursors are waves propaga- 

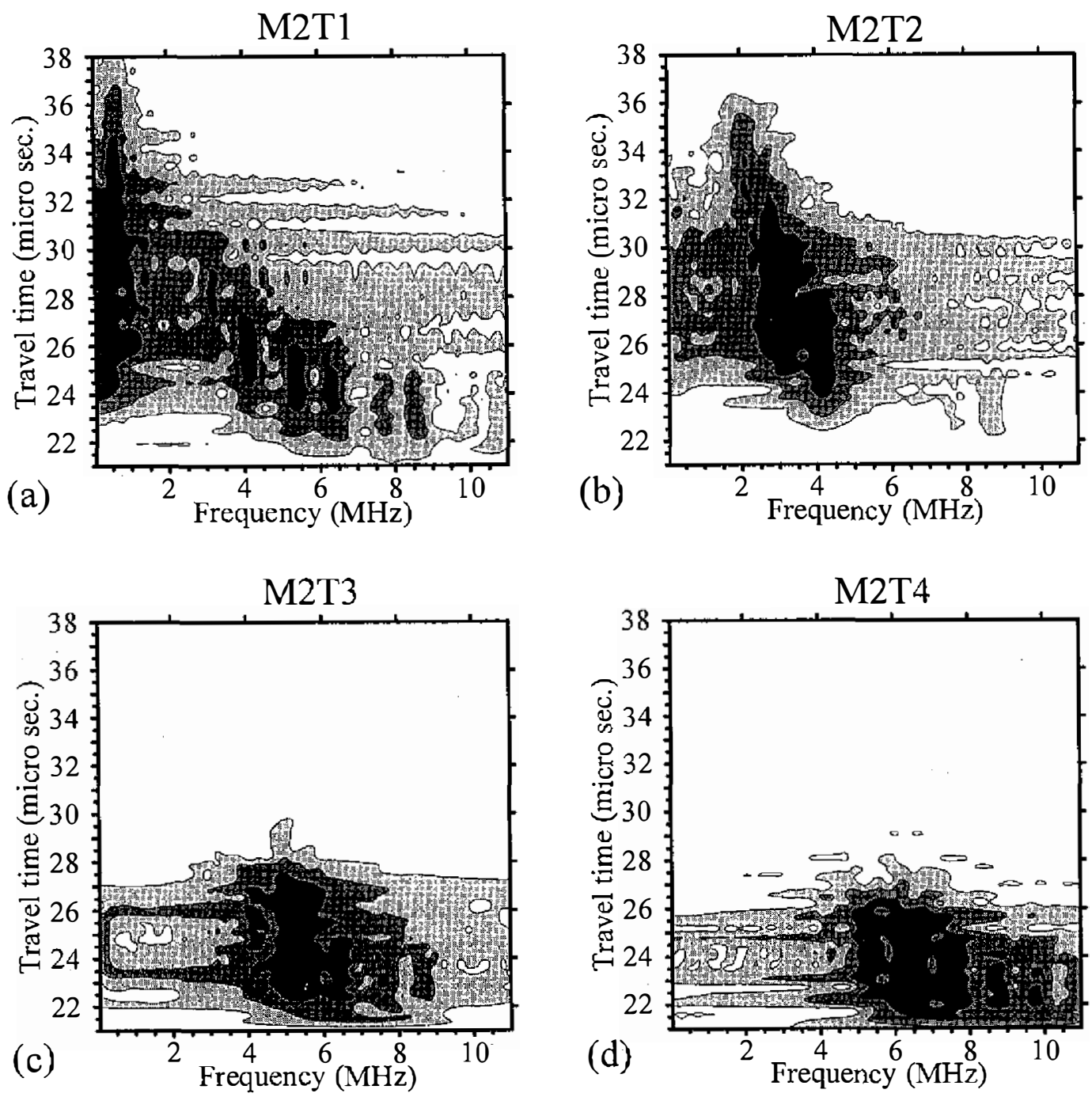

Fig. 7. The dispersion curves of the precursors recorded for the Model M2. The signals recorded by (a) $1 \mathrm{MHz}$ (b) $2.5 \mathrm{MHz}$ (c) $5 \mathrm{MHz}$ (d) $10 \mathrm{MHz}$ transducers.

tion within a thin HVL and refracted to the receivers (e.g., Smith et al. 1994). The low-frequency waves, which can be predicted by the Jeffreys-Bullen travel time table, are the waves that propagate within the normal mantle. According to the results of our experiments, the high-frequency and high-velocity precursors can exist within a thin HVL and can be refracted to the receivers. The low-frequency and low-velocity waves propagate in the low-velocity master material (epoxy). The early high-frequency precursors are dispersive with high-frequencies arriving early.

Stuart et al. (1995) analyzed the broadband seismic data and found that high frequencies 
arrive early and that below $5 \mathrm{~Hz}$ there is dispersion to $1 \mathrm{~Hz}$. The curvatures of the dispersion curves of the early high-frequency precursors are different for the different thickness HVLs (Figs. 8 and 9) in our study. Therefore, the curvature of the dispersion curve of the early highfrequency precursors may be used to estimate the thickness of the HVL. Holes (the local low values) can be found in the dispersion curves of the early high-frequency precursors (Gubbins and Snieder 1991; Stuart et al. 1995), and can also be revealed in Figs. 8 and 9. If the period of the holes can be observed in the field data, the thickness of the HVL may be evaluated using the period of the exciting mode of the early high-frequency precursors.
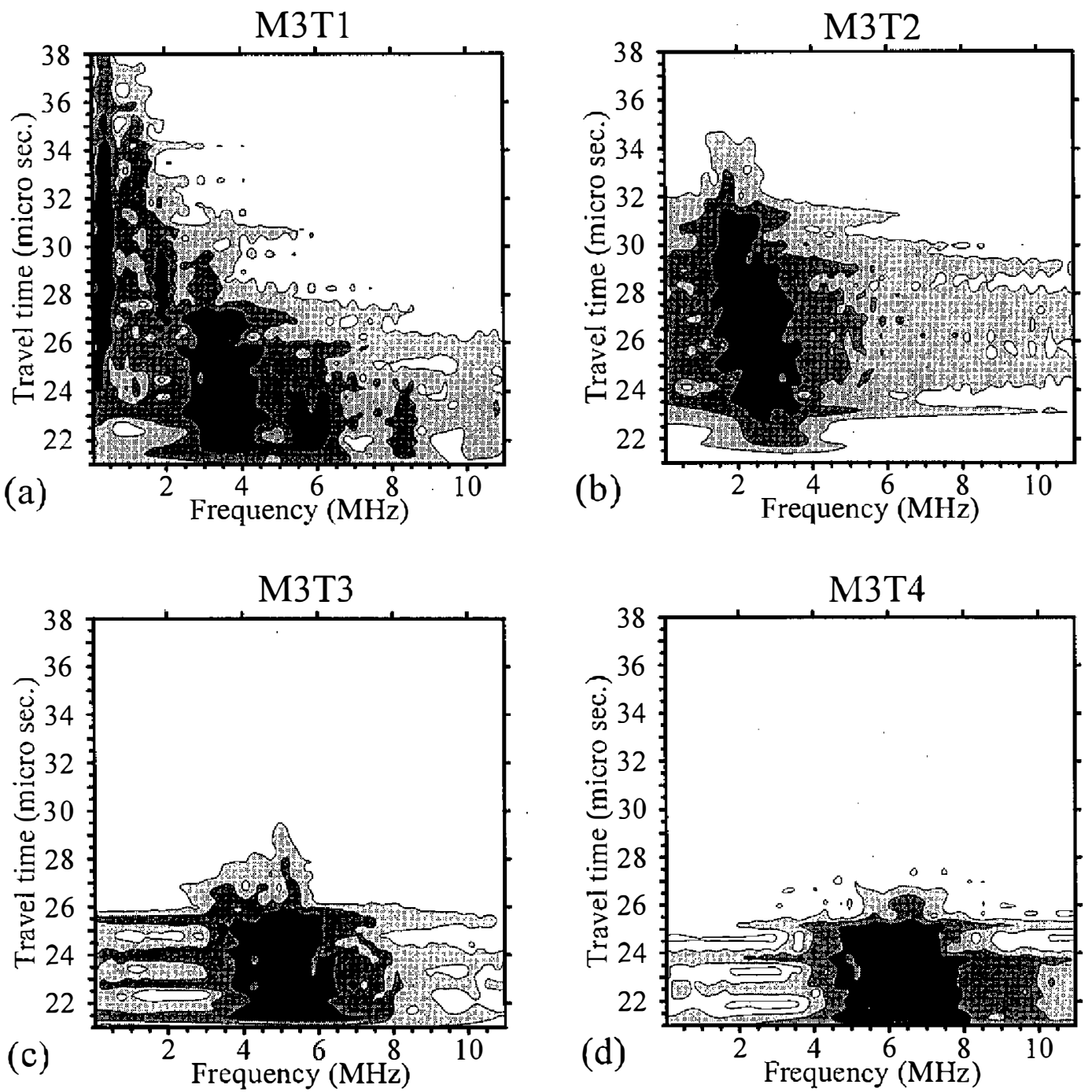

Fig. 8. The dispersion curves of the precursors recorded for the Model M3. The signals recorded by (a) $1 \mathrm{MHz}$ (b) $2.5 \mathrm{MHz}$ (c) $5 \mathrm{MHz}$ (d) $10 \mathrm{MHz}$ transducers. 


\section{Receiver: T5, S-type transducer Source: $\mathbf{T 1}$}

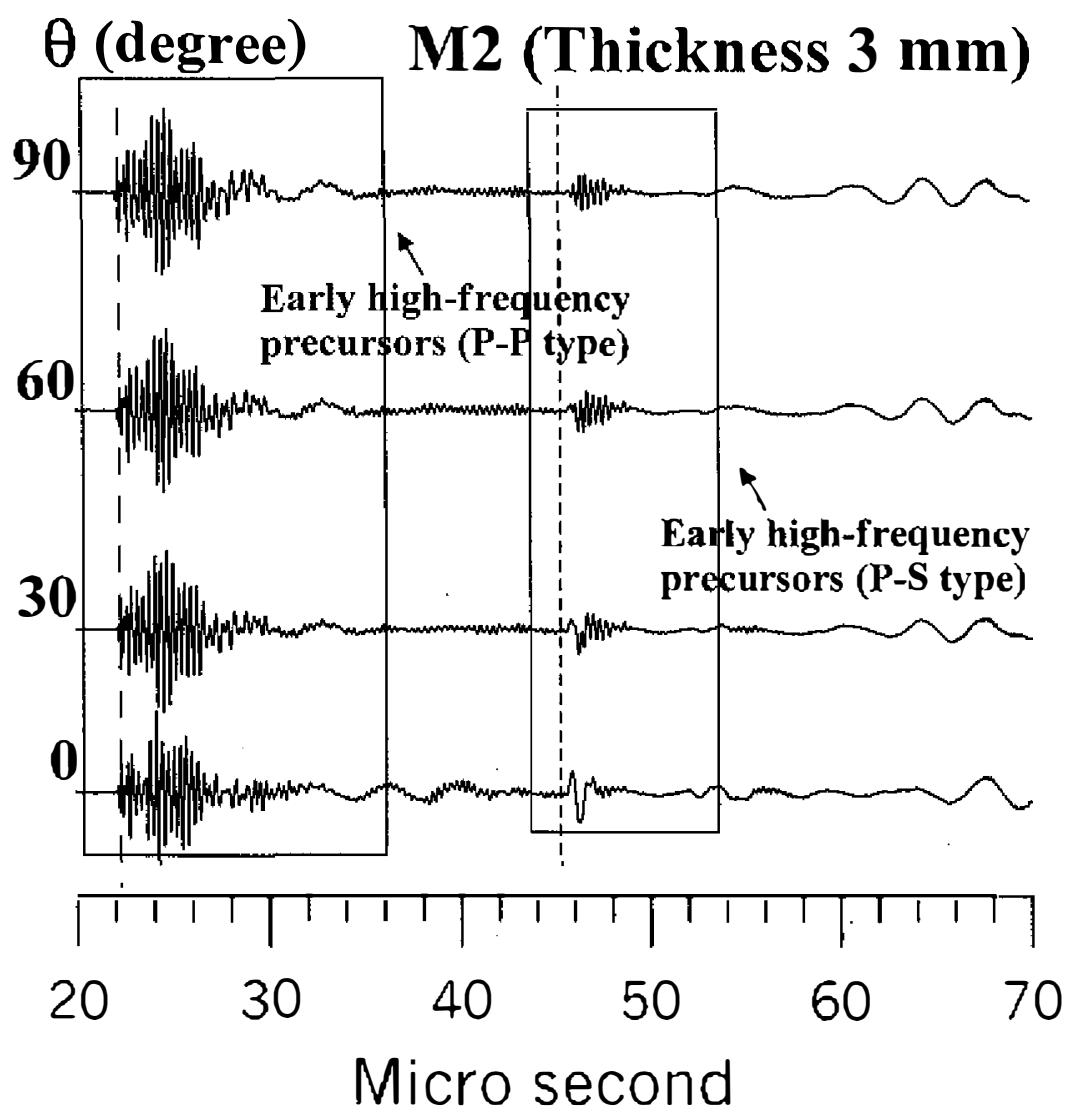

Fig. 9. The signals detected by the shear wave receiver (T5) for the Model M2. $\theta$ is the angle between the polarization of the S-type transducer and the duralumin plate. The long and short slashed lines are the arrival times of $\mathrm{P}$ and $\mathrm{S}$ waves propagation at $\mathrm{P}$ and $\mathrm{S}$ wave velocities in the thin duralumin plate, respectively.

\section{CONCLUSIONS}

Although not all the earth structures can be satisfyingly simulated in this experimental study, some of the essential attributes of the precursory phases can still be obtained.

From the experimental results, it was observed that the high-frequency and high-velocity dispersive precursors can propagate in the thin HVL models, and that high-frequency waves arrive early. The thinner the HVL, the smaller the amplitude and higher frequency of the precursors are. 
P-to-S early high-frequency precursors were observed in this study, but the their amplitude was noted to be smaller than the P-to-P precursors. Based on the arrival times and amplitudes of the precursors and the geometry of the receiver stations relative to the HVL, the precursors are the $\mathrm{P}$ waves radiated from the source and refracted into the HVL, propagating in the HVL and leaking from the HVL to the receiver. The leading role in controlling the propagation of precursors is played by the P-to-P interactions.

The early high-frequency precursors are the resonant waves propagation in a thin HVL, and the resonant wavelengths of the waves are equal to $2 \mathrm{t} / \mathrm{n}$, where $\mathrm{n}$ is an integer indicating the order of the harmonic and $t$ is the thickness of the slab. The curvatures of the dispersion curves and the period of the holes in the dispersion curves of the early high-frequency precursors are different for the different thickness HVLs. Therefore, the curvature of the dispersion curve and the period of the holes in the dispersion curve of the early high-frequency precursors may be used to estimate the thickness of the HVL.

Acknowledgement The support of this study by the National Science Council of Taiwan, R. O. C. under Contract No. NSC892116M194012 is deeply appreciated.

\section{REFERENCES}

Ansell, J. H., and D. Gubbins, 1986: Anomalous high-frequency wave propagation from the Tonga-Kermadec seismic zone to New Zealand. Geophys. J. R. Astron. Soc., 85, 93106.

Blitz, J., and G. Simpson, 1996: Ultrasonic methods of nondestructive testing. Chapman \& Hall, London.

Chiu, J-. M., B. L. Isacks, and R. K. Cardwell, 1985: Propagation of high-frequency seismic waves inside the subducted lithosphere from intermediate-depth earthquakes recorded in the Vanuatu arc. J. Geophys. Res., 90, 12,741-12,754.

Galea, P., 1992: Observations of very high P-velocities in the subducted slab, New Zealand, and their relation with the slab geometry. Geophys. J. Int., 110, 238-250.

Gubbins, D., and R. Snieder, 1991: Dispersion of P waves in subducted lithosphere: evidence for an Eclogite layer. J. Geophys. Res., 96, 6321-6333.

Gubbins, D., A. Barnicoat, and J. Cann, 1994: Seismological constraints on the gabbro-eclogite transition in subducted oceanic crust. Earth and Planetary Science Letters, 122, 89101.

Van der Hilst, R., and R. Snieder, 1996: High-frequency precursors to $\mathrm{P}$ waves arrivals in New Zealand: implications for slab structure. J. Geophys. Res., 101, 8473-8488.

Huppert, L. N., and C. Frohlich, 1981: The P velocity within the Tonga Benioff zone determined from traced rays and observations. J. Geophys. Res., 86, 3771-3782.

Shin, T. C., 1992: Some Implications of Taiwan Tectonic Features from the Data Collected by the Central Weather Bureau Seismic Network. Meteorological Bulletin, 38, 23-48.

Smith, G. P., D. Gubbins, and W. Mao, 1994: Fast P waves propagation in subducted Pacific lithosphere: refraction from the plate. J. Geophys. Res., 99, 23787-23800. 
Stuart, G. W., D. Francis, D. Gubbins, and G. Smith, 1995: Tararua broadband array, North Island, New Zealand. Bull. Seismol. Soc. Am., 85, 325-333. 